\title{
Biotyping of Multidrug Resistant Campylobacter Jejuni from Poultry and Humans in Northern Region of Ghana
}

\section{Akosua Bonsu Karikari ( $\square$ asbuks@yahoo.co.uk )}

University for Development Studies

Courage Kosi Setsoafia Saba

University for Development Studies

Stephen Wilson Kpordze

University for Development Studies

Research article

Keywords: Campylobacter, Antibiotic resistance, Multidrug resistance, Biotyping, Ghana

Posted Date: July 6th, 2020

DOI: https://doi.org/10.21203/rs.3.rs-38356/v1

License: (c) This work is licensed under a Creative Commons Attribution 4.0 International License.

Read Full License 


\section{Abstract}

\section{Background}

Thermophilic Campylobacters are zoonotic bacteria which are universally famous for causing enteritis in humans. They are normally found as commensals in the digestive tract of food animals with poultry being a major reservoir of the pathogenic species. This study evaluated the presence of Campylobacter in poultry (commercial and domestic) and humans (patients and asymptomatic individuals) and characterized strains by biotyping and susceptibility test in the Northern region of Ghana.

\section{Results}

Of the 346 poultry and 741 human samples analyzed, $43.1 \%$ and $12.9 \%$ Campylobacter species were recovered. Isolation frequency from commercial birds was significantly more (50.5\%) than domestic birds (33.8\%), as incidence in patients and asymptomatic individuals was $15.4 \%$ and $9 \%$ with a $p=0.001$. Campylobacter jejuni were recovered from more than eighty percent (84\%) of commercial birds and $64 \%$ of domestic birds and in humans significantly less strains were observed in patients than asymptomatic individuals $(p<0.05)$. Distribution of biotypes in domestic birds, patients and asymptomatic individuals were comparable with $C$. jejuni biotype I prevalence and Bioytype II largely in commercial poultry. All strains were resistant to tetracycline; and against erythromycin, $69.3 \%$ of jejuni strains were resistant but no resistance was recorded among the non-jejuni strains. Most resistance was noted among patient strains. Imipenem and the aminoglycosides were relatively effective as resistance of $10 \%$ and below $20 \%$ were obtained. Multidrug resistance was between $94-100 \%$ with $60 \%$ of strains being resistant to 4 or 5 classes of antibiotics.

\section{Conclusion}

This study found phenotypic correlation between poultry and human strains coupled with copious isolation of $C$. jejuni biotypes I and II from commercial and domestic poultry which may well be possible source of threat to public health. None of the endorsed treatment drugs (erythromycin, ciprofloxacin, and tetracycline) can be admitted in this region due to common resistance found among strains against these agents but imipenem and aminoglycosides proved to be reliable as strains showed ample susceptibility. The multiple drug resistance (94-100\%) observed in our study and other related investigations in the country call for rapid implementation of the policy on antimicrobial use and resistance which has been launched in Ghana.

\section{Background}

Campylobacter are Gram-negative zoonotic pathogens of global significance, especially the thermophilic species. Of the three species (jejuni, coli, lari), Campylobacter jejuni is crucial and liable for colonic 
colonization in poultry and food-borne enteritis in humans. Generally, $C$. jejuni is non-pathogenic in poultry though freshly hatched chickens are susceptible to ephemeral diarrhea following infection. Consumers of undercooked poultry meat, beef, pork and poultry products may be at risk of campylobacteriosis which is a major cause of human enteritis [1].

The importance of campylobacteriosis as a foodborne pathogen is established by surveillance studies reporting 15918 hospitalizations in the UK [2], 2, 00,507 cases in the European Union [3] and an incidence of 12.71 cases per 100.000 inhabitants in USA [4]. Inadequate systematic investigations to detect outbreaks whose strains could serve as basis for source attribution and risk valuation make it hard to establish factual incidence of the infection in developing countries [5]. But campylobacteriosis is viewed as hyper-endemic in many developing regions because of numerous factors including poor environmental sanitation, proximity with animals at native settings in rural, peri urban and farming communities [6]. Marquis and colleagues recount live, unrestricted birds kept in close association with humans as key potential source of $C$. jejuni infection [7]. Likewise in the UK, $97 \%$ of sporadic infections have been linked to farmed animals [8]. The degree to which poultry intake is responsible for human infections is however not precisely known [9].

Resistance trends and biotyping have been used in epidemiological investigations to track potential sources or supposed routes of campylobacteriosis outbreaks. Such studies have shown prevalence of different biotypes of species oscillating between $40-100 \%$ in birds including chicken $[10,11]$. Semblance of Campylobacter species recovered from human patients and poultry proposes commonality of infection according to Bruce et al. [12]. This has been validated by some studies which have shown related types of Campylobacter from poultry and humans $[13,14]$.

Antimicrobial therapy in campylobacteriosis is generally recommended in severe cases and immunesuppressed individuals in which case macrolides (first choice) and fluoroquinolones (second choice) are considered [15]. These drugs were previously reported to be effective but misuse of antibiotics in both veterinary and human medicine have contributed to escalated incidence of resistant strains documented worldwide [16].

In evaluating the threat associated with the presence of Campylobacter in poultry, it is an essential step to identify phenotypic characters that provide epidemiological markers in both animal and human population. The Northern region of Ghana is an agricultural region with majority of the people engaged in animal husbandry but research on zoonotic infections including campylobacteriosis is dolefully inadequate. To provide a baseline information on this important pathogen, the study determined the presence of Campylobacter in the cloaca of poultry and the stools of symptomatic and asymptomatic individuals, assessed the resistance patterns of species and compared the biotypes of $C$. jejuni in humans and poultry.

\section{Results}

Prevalence of Campylobacter in poultry and humans 
Campylobacterwas present in $43.1 \%$ of poultry $(149 / 346)$ and $12.9 \%$ in humans $(96 / 741)$ and the difference was significant. In the commercial birds, prevalence was $50.5 \%(97 / 192)$ as $33.8 \%(52 / 154)$ was found in the domestic birds with a $p=0.001$. In humans, rates of $15.4 \%(71 / 462)$ and $9 \%(25 / 279)$ were recorded respectively among patients and asymptomatic individuals, Table 1.

Table 1

Occurrence of Campylobacter in poultry and humans in Northern Ghana

\begin{tabular}{|lllll|}
\hline \multicolumn{5}{|c|}{ Frequency (\%) } \\
\hline Sample Source & No. of Sample & No. of Positive & No. of Negative & P-value \\
\hline Poultry & 346 & $149(43.1)$ & $197(56.9)$ & \\
Humans & 741 & $96(12.9)$ & $645(87.0)$ & \\
\hline Total & 1087 & $245(22.5)$ & $842(77.5)$ & 0.001 \\
Commercial poultry & 192 & $97(50.5)$ & $95(49.5)$ & \\
\hline Domestic poultry & 154 & $52(33.8)$ & $102(66.2)$ & \\
\hline Total & 346 & $149(43.1)$ & $197(56.9)$ & 0.001 \\
\hline Patients & 462 & $71(15.4)$ & $391(84.6)$ & \\
Asymptomatic individuals & 279 & $25(9.0)$ & $254(91.0)$ & \\
\hline Total & 741 & $96(12.9)$ & $645(87.0)$ & 0.001 \\
\hline
\end{tabular}

Occurrence of Campylobacter jejuni species in poultry and humans

Out of the 245 Campylobacter isolates confirmed by latex agglutination, only 105 were further characterized with a distribution as follows; 75 poultry strains (50 commercial, 25 domestic) and 30 human isolates (20 patients, 10 asymptomatic). Campylobacter jejuni were found in $84 \%(42 / 50)$ of commercial poultry, $64 \%(16 / 25)$ of domestic poultry, $45 \%(9 / 20)$ in patients and $50 \%(5 / 10)$ in asymptomatic individuals with a statistically significant difference $(P=0.001)$, Fig. 1.

\section{Distribution of Campylobacter jejuni biotypes in poultry and humans}

The distribution of Biotypes revealed Biotype I dominance in asymptomatic individuals $(80 \% ; 4 / 5)$, patients $(66.7 \% ; 6 / 9)$ and domestic poultry $(56.3 \% ; 9 / 16)$ as Biotype II was prevalent in commercial poultry $(59.5 \% ; 25 / 42)$ but none among patient strains. Biotype III was mainly found in patients $(33.3 \%$; $3 / 9)$ and commercial poultry $(14.3 \% ; 6 / 42)$ but $0 \%$ in asymptomatic individuals. Biotype IV was only isolated from poultry, one (1) isolate each from commercial and domestic sources. Overall, Biotype I was more common among human strains $(71.4 \%, 10 / 14)$ as Biotype II were to poultry $(51.7 \%, 30 / 58)$, Table 2 . 
Table 2

Biotypes of Campylobacter jejuni from poultry and humans

\begin{tabular}{|lllllll|}
\hline Sources & No. of isolates & \multicolumn{2}{l}{ Percentage frequency (\%) } & P value \\
\cline { 3 - 6 } & & Biotype-I & Biotype-ll & Biotype-III & Biotype-IV & \\
\hline Poultry & & & & & & \\
Commercial & 42 & $10(23.8)$ & $25(59.5)$ & $6(14.3)$ & $1(2.4)$ & \\
Domestic & 16 & $9(56.3)$ & $5(31.3)$ & $1(6.3)$ & $1(6.3)$ & \\
Total & 58 & $19(32.8)$ & $30(51.7)$ & $7(12.1)$ & $2(3.4)$ & 0.170 \\
Humans & & & & & & \\
Patients & 9 & $6(66.7)$ & 0 & $3(33.3)$ & 0 & \\
Asymptomatic & 5 & $4(80.0)$ & $1(20.0)$ & 0 & 0 & 0.084 \\
\hline Total & 14 & $10(71.4)$ & $1(7.1)$ & $3(21.4)$ & 0 & \\
\hline
\end{tabular}

\section{Resistance of Campylobacter species in poultry and humans}

All isolated Campylobacter strains were resistant to tetracycline and to erythromycin, resistance of 60$90 \%$ was recorded. The $\beta$-lactam drugs, ampicillin and ceftriaxone performed poorly with $78-95 \%$ resistance recorded. Among the fluoroquinolones, norfloxacin was most effective (12-30\%), followed by nalidixic acid (20-45\%) but relatively high resistance was observed against ciprofloxacin (48-70\%). Highest susceptibility of strains were recorded in aminoglycosides and imipenem (100-80\%). In general, patient strains showed significantly higher resistance $(p=0.001)$, Fig. 2.

\section{Resistance of Campylobacter jejuni and non-jejuni species}

All $(100 \%)$ the non- jejuni strains were susceptible to erythromycin while $69.4 \%$ of $C$. jejuni were resistant but against trimethoprim sulphamethoxazole (SXT), nalidixic acid and chloramphenicol, non-jejuni strains showed more resistance which was significant, $P=0.001$. Drugs which recorded more $C$. jejuni resistance included ciprofloxacin, norfloxacin, amikacin and imipenem, Fig. 3.

\section{Resistance pattern of Campylobacter jejuni biotypes in poultry and humans}

Most resistance to erythromycin (82.8\%) was found in Biotype I. Biotype II strains generally showed lower resistance, with exception to ciprofloxacin and norfloxacin where their resistance were more. Resistance of Biotype II to chloramphenicol was below $10 \%$ but up to $52 \%$ rate was recorded among Biotypes I and III strains. Highest resistance to trimethoprim sulphamethoxazole was observed among Biotype III strains, Fig. 4. The only two isolated strains of Biotype IV were resistant to ceftriaxone but susceptible to erythromycin, nalidixic, norfloxacin, imipenem and the aminoglycosides. With the exception of chloramphenicol, resistance difference among biotypes was not statistically significant. Multidrug 
resistance was $94.7 \%$ in poultry and $100 \%$ among human strains. Sixty percent $(60 \%)$ of poultry and human strains were resistant to 4 or 5 classes and up to about $37 \%$ to 6 or 7 classes of antibiotics, Table 3.

Table 3

Multidrug resistance pattern exhibited by poultry and human strains

\begin{tabular}{|lll|}
\hline Multidrug Resistance & Poultry & Humans \\
\cline { 2 - 4 } & No. of Resistant strains (\%) & $\begin{array}{l}\text { No. of Resistant strains } \\
(\%)\end{array}$ \\
\hline 3 classes of antibiotics & $16(24.6 \%)$ & $2(6.7 \%)$ \\
\hline 4 classes of antibiotics & $23(35.4 \%)$ & $10(33.3 \%)$ \\
\hline 5 classes of antibiotics & $16(24.6 \%)$ & $8(26.7 \%)$ \\
\hline 6 classes of antibiotics & $8(12.3 \%)$ & $4(13.3 \%)$ \\
\hline 7 classes of antibiotics & $2(3.1 \%)$ & $5(16.7 \%)$ \\
\hline 8 classes of antibiotics & - & $1(3.3 \%)$ \\
\hline Total & $65(100 \%)$ & $30(100 \%)$ \\
\hline
\end{tabular}

\section{Discussion}

Considering potential risks associated with Campylobacter in poultry, it is critical to identify these strains in the human population. This study isolated and characterized Campylobacterfrom poultry and humans and respectively found prevalence of $43.1 \%$ and $12.9 \%$ which is higher in the case of poultry but slightly lower in reference to human, than reported rates in the southern region of the country $[17,18]$. Elsewhere in Canada, comparable rate of $40 \%$ was reported in poultry while divergent results of $62 \%, 87.2 \%$ and $90 \%$ were recorded in Morocco, Poland and Cameroon [14, 19-21]. A review of most studies across Africa reporting on Campylobacter isolation from stools generally documented low rates [22], which could be attributed to the isolation method that is typically culture rather than PCR which is superior and more sensitive in detection. Recovery of Campylobacter from patients with enteritis is usually greater than asymptomatic individuals as shown in our study where $15.4 \%$ and $9 \%$ were respectively recorded with supporting reports of $55 \%$ rate among patients in Nigeria, $16.6 \%$ from individuals in contact with foodproducing animals in central Egypt and 11.4\% from individuals in eastern Tanzania [23-25].

Campylobacter jejuni dominance (80\%) in poultry was normal, conforming to literature reports as the most common species found in poultry and its products. In humans however, more non-jejuni species were recovered and the explanation could be the possible acquisition from different sources such as other animals (livestock) and the environment where these non-jejuni strains are rifer. It was intriguing to find more non-jejuni strains in the domestic birds than the commercial birds which could be credited to 
the free access of domestic birds to the environment and other animals where they could have picked the strains.

Biotyping of $C$. jejuni was explored to reveal the relatedness of poultry and human strains and to provide epidemiological indicators in the study of Campylobacterinfections in this region. The distribution of biotypes in patients, asymptomatic individuals and domestic birds were similar with Biotype I incidence while analogous findings have been described in Australia, France, Iran and Nigeria [13,26-28]. A study by Nadeau and colleagues discovered almost all invasive strains of $C$. jejuni belonged to biotypes I and II as cell-cytotoxic isolates were associated with III and IV and further suggested that clonally related isolates have common in vitro virulence characteristics [29]. In this study, the phenotypic relationship between poultry and human strains as well as the high proportion of $C$. jejuni biotypes I and II in both commercial and domestic poultry is a potential source of threat for humans as infections from these biotypes could be invasive and consequently dire. But, further studies on virulence factors and an assessment of the outcomes of infections caused by these biotypes would give a better understanding of this risk, since invasive properties of C. jejuni strains can influence the austerity of clinical changes [1].

Routine treatment for Campylobacterinfection is erythromycin but fluoroquinolones (specifically ciprofloxacin) and tetracycline are alternatively prescribed. Susceptibility to tetracycline was $0 \%$ in both poultry and human strains. This results was not startling because tetracycline which is an over the counter drug is readily available and among the popular drugs consumed in both veterinary and clinical practice in Ghana. It is frequently used by commercial poultry farmers and well identified by rural domestic breeders as prophylaxis and treatment drug for poultry infections. It is also habitually prescribed in hospitals to manage enteritis and other conditions (Personal communication).

Resistance to erythromycin was only found in $C$. jejuni as $100 \%$ susceptibility was detected among nonjejuni strains. Although some countries have recorded low and stable rates of resistance to erythromycin $[30,31]$ studies in Ghana and some other African countries have observed contradictory results [32, 33, $17,18]$. Erythromycin as a drug of choice has performed poorly against human and animal Campylobacters in Ghana as evidenced in previous studies in a different region of the country $[17,18]$. Some authors have predicted increased risk of adverse events including Guillain-Barré syndrome and severe reactive illnesses with macrolide-resistant Campylobacters [34]. Other investigations also predict an association of macrolide resistance in clinical $C$. jejuni strains with some virulence markers and so the use of it in any ecology may select for such strains [34]. Thus, occurrence of macrolide resistant Campylobacter could be of public health significance even in the absence of proof of treatment failure [35]. The carbapenems (meropenem, imipenem) are among the last line drugs often used in the management of infections caused by multidrug resistant pathogens in Ghanaian hospitals (Personal communication). High susceptibility of up to $100 \%$ were previously reported $[17,18]$ but recent studies indicate increasing resistance though not at alarming levels (10\%).

Multidrug resistance was $94.7 \%$ in poultry and $100 \%$ in humans with $60 \%$ of isolates showing resistance to four or five drugs. Multiple drug resistance invariably leads to expensive health care cost with 
attendant financial drain particularly on the poor and average patient in a Low Middle Income Country. The fallout of antimicrobial resistance in animals and humans is an emphasis of commonly used and abused drugs. This became evident in our study where resistance of $10 \%$ and below $20 \%$ were observed against imipenem and the aminoglycosides. Because these drugs are used to manage severe and complicated infections they are infrequently prescribed and also not readily available for potential abuse.

In view of these, antibiotic resistance surveillance studies in animal, human and environment have become obligatory especially in under resourced regions of the country as most studies are focused in the well-resourced regions. Data from such investigations will inform stakeholders and buttress the need for the implementation of the national policy on antimicrobial use and resistance.

\section{Conclusion}

The study found phenotypic linkages between poultry and human strains with $C$. jejuni Biotype I and II dominance, that might pose some threat to human through consumption of contaminated poultry to which further studies are recommended to establish the extent of risk. All isolated Campylobacter species were resistant to tetracycline as patient strains were commonly resistant. None of the endorsed treatment drugs (Erythromycin, tetracycline and ciprofloxacin) for Campylobacter infections may be empirically safe in this region without susceptibility confirmation from the laboratory. The carbapenems (Imipenem) and the aminoglycosides (amikacin, gentamicin) on the other hand appeared effective in-vitro. The recorded MDR of $95-100 \%$ re-echo the urgency to implement the national policy on antimicrobial use and resistance before the situation becomes unmanageable.

\section{Methods}

\section{Study Area}

This research was conducted in the Tamale metropolis and Tolon district of the Northern Region. Tamale, the capital of the Region is the third largest city in Ghana and located $600 \mathrm{~km}$ north of Accra, the capital of the country. Sampling sites included the Tamale Teaching Hospital (TTH), Tamale Central Hospital (TCH), six (6) commercial poultry farms in Tamale metropolis and households which reared domestic poultry in the Tolon district. The Tamale Teaching Hospital is an 800 bed capacity tertiary care facility. It provides referral services to the three Regions in the northern sector of Ghana. The Tamale Central Hospital is a 186 bed capacity secondary care facility which supports the teaching hospital in health care services.

\section{Sampling}

A total of 1087 samples were screened from 25th October, 2017 to 7th May, 2019. In all, 462 stool samples were collected from patients in both hospitals, 279 stool samples from asymptomatic individuals from Tolon district, 192 and 154 cloacal swabs (eswab Copan Italia) respectively from 
domestic and commercial chicken. Stool containers were given to household individuals who raised poultry in their homes and consented to be part of the study. All samples were kept on ice packs in an insulator box and transported within 2 hours to the laboratory for analysis.

\section{Processing and isolation of Campylobacter species}

A loopful of fresh faeces was cultured directly on modified charcoal-cefoperazone-deoxycholate agar (mCCDA Oxoid CM0689) supplemented with CCDA selective- supplement (Oxoid SRO155E). Cloacal swabs were plated directly on mCCDA agar and kept in a $2.5 \mathrm{~L}$ anaerobic jar containing Campy-Gen gas generating kit (Oxoid CN0025A) to keep the microaerobic condition. Incubation was at $42^{\circ} \mathrm{C}$ for 48 hours. Plates which showed no growth after 48hrs were declared negative.

\section{Identification And Confirmation Of Isolates}

Colonies typical of Campylobacter species on mCCDA agar were picked and streaked onto blood agar ((Oxoid, Basingstoke, UK) supplemented with $5 \%$ sheep blood, incubated microaerobically at $42^{\circ} \mathrm{C}$ for $24 \mathrm{hrs}$. Gram stain, catalase and oxidase test were performed on all suspected colonies. Isolates were confirmed by latex agglutination test (Oxoid, Basingstoke, UK) following manufacturer's instructions. The hippurate test kit (Sigma-Aldrich, 01869) was used to categorize confirmed isolates into jejuni and non jejuni strains. Isolates were stored in cryo vials containing brain heart infusion broth (BHI) with $15 \%$ glycerol at $-20^{\circ} \mathrm{C}$ prior to susceptibility test and biotyping. Campylobacter isolates from previous studies $[17,18]$ were used as controls in this study.

\section{Biotyping of Campylobacter jejuni strains}

The $C$. jejuni isolates were typed using the Lior's scheme where strains are characterized based on hippurate hydrolysis, rapid H2S production and DNase test (Lior, 1984). Hippurate hydrolysis was performed using hippurate test kit (Sigma-Aldrich, 01869) and the development of a blue-purple colour indicated a positive reaction. A positive DNase reaction revealed a large, clear zones of hydrolysis around a colony on DNase agar plate (Oxoid, UK). Hydrogen sulphide positive strains exhibited blackening in the test medium (Oxoid, UK, CM0277).

\section{Antimicrobial Susceptibility Test}

The Kirby-Bauer disk diffusion test was carried out on 250 Campylobacter strains using the following antibiotics (Oxoid, Basingstoke, UK): Ampicillin $(10 \mu \mathrm{g})$, trimethoprim sulphamethoxazole $(25 \mu \mathrm{g})$, ciprofloxacin $(5 \mu \mathrm{g})$, erythromycin $(15 \mu \mathrm{g})$, gentamicin $(10 \mu \mathrm{g})$, nalidixic acid $(30 \mu \mathrm{g})$, chloramphenicol $(30 \mu \mathrm{g})$, ceftriaxone $(30 \mu \mathrm{g})$, norfloxacin $(10 \mu \mathrm{g})$, amikacin $(30 \mu \mathrm{g})$, imipenem $(10 \mu \mathrm{g})$ and tetracycline $(30 \mu \mathrm{g})$. Saline suspension of $0.5 \mathrm{Mc}$ Farland standard was inoculated on Mueller-Hinton agar (Oxoid, UK) supplemented with $5 \%$ sheep blood, incubated at $42{ }^{\circ} \mathrm{C}$ for 24 hours under microaerobic condition using CampyGenTM 2.5L, Oxoid. Susceptibility results were interpreted using EUCAST guidelines (EUCAST, 
2019) for Campylobacter, but breakpoints for Enterobacteriaceae was adopted for antibiotics yet to have. Multidrug resistance was defined as resistance to three or more classes of antibiotics.

\section{Data Analysis}

Data was entered into Microsoft Excel and analyzed in IBM SPSS version 20. Results were presented in tables and graphs. Descriptive statistics as frequencies and percentages were used. Associations between categorical outcome variables were calculated by Fisher's exact test and a P-value less than 0.05 was considered significant.

\section{Abbreviations}

MDR-Multidrug resistance

TCH-Tamale Central Hospital

TTH-Tamale Teaching Hospital

BHI- Brain Heart Infusion

\section{Declarations}

\section{Ethics approval and consent to participate}

The Research Ethical Review Committee of the Tamale Teaching Hospital gave authorization (TTHERC/19/06/18/03) for the study. Verbal consent was sought from patients, commercial poultry farmers and individuals in the various households in the Tolon district.

\section{Consent for publication}

Not applicable

\section{Availability of data and materials}

We consider our data private but the corresponding author will make it available upon reasonable request.

\section{Competing interests}

The authors declare that they have no competing interests

\section{Funding}

Financial support was received from Faculty of Science, University of Helsinki for sample collection. 


\section{Authors' contributions}

All authors read and consented to the final draft of the manuscript. ABK was involved in the conception, study design and drafting of the manuscript, CKSS contributed in conception and drafting of the manuscript, SWK was involved in sample collection and processing as well as data analysis

\section{Acknowledgements}

Our thanks go to the laboratory staff of TTH and TCH for the assistance in patient sample collection, commercial poultry farm owners in the Tamale metropolis for allowing us to take cloacal swabs from their birds as well as individuals who agreed to be part of this study. We are also grateful to Dr. Kaisa Haukka of the University of Helsinki, Finland for her role in sourcing funds for the sample collection.

\section{References}

1. Shane SM. The significance of Campylobacter jejuni infection in poultry: A review. Avian Pathol. 1992;21(2):189-213.

2. Adak GK, Meakins SM, Yip H, Lopman BA, O' Brien SJ. Disease risks from foods, England and Wales, 1996-2000. Emerg Infect Dis. 2005;11:365-72.

3. European Food Safety Authority (EFSA). The community summary report on trends and sources of zoonoses and zoonotic agents in the European Union in 2007. EFSA J. 2009;223:1-217.

4. Centers for Disease Control and Prevention (CDC). Preliminary FoodNet data on the incidence of infection with pathogens transmitted commonly through food - 10 States, 2006. Morbid Mortal Wkly Rep. 2007;56:336-9.

5. World Health Organisation (WHO). The global view of Campylobacteriosis. World Health Organization: Geneva, Switzerland. 2013; p. 69.

6. Samuel SO, Aboderin AO, Akanbi AA, Adegboro B, Smith SI, Coker AO. Campylobacter enteritis in Ilorin, Nigeria. East Afr Med J. 2006;83:478-84.

7. Marquis GS, Ventura G, Gilman RH, Porras E, Miranda E, Carbajal L, Pentafiel M. Fecal contamination of shanty town toddlers in households with non-corralled poultry, Lima, Peru. Am J Public Health. 1990;80:146-9.

8. Wilson DJ, Gabriel E, Leatherbarrow AJH, Cheesbrough J, Gee S, Bolton E, Fox KA, Hart CA, Diggle PJ, Fearnhead $\mathrm{P}$. Rapid evolution and the importance of recombination to the gastroenteric pathogen Campylobacter jejuni. Mole Biol Evol. 2009;26(2):385-97.

9. Jacobs-Reitsma W. Campylobacter in food supply. In: Nachamkin I, Blaser MJ, editors. Campylobacter. 2nd ed. Washington, D.C.: American Society for Microbiology; 2000. pp. 467-81.

10. Ridsdale JA, Atabay HI, Corry JEL. Prevalence of Campylobacters and Arcobacters in ducks at the abattoir. J Appl Microbiol. 1998;85:567-73. 
11. Dickins MA, Franklin S, Stefanova R, Schutze GE, Eisenach KD, Wesley I, Cave MD. Diversity of Campylobacterisolates from retail poultry carcasses and from humans as demonstrated by pulsedfield gel electrophoresis. J Food Prot. 2002;65:957-62.

12. Bruce D, Zochowski W, Ferguson LR. Campylobacter enteritis. Br Med J. 1977;2:1219-20.

13. Shanker S, Rosenfield JA, Davey GA, Sorrell TC. Campylobacter jejuni: Incidence in broilers and biotype distribution in human and broiler isolates. Appl Environ Microbiol. 1982;43:1219-20.

14. Nadeau É, Messier S, Quessy S. Prevalence and comparison of genetic profiles of Campylobacter strains isolated from poultry and sporadic cases of Campylobacteriosis in humans. J Food Prot. 2002;65:73-8.

15. World Health Organisation (WHO). WHO estimates of the global burden of foodborne diseases; WHO: Geneva, Switzerland, 2015; p. 268.

16. lovine NM. Resistance mechanisms in Campylobacter jejuni. Virulence. 2013;4:230-40.

17. Karikari AB, Obiri-Danso K, Frimpong EH, Krogfelt KA. Multidrug resistant Campylobacter in faecal and carcasses of commercially produced poultry. Afr J Microbiol Res. 2017a;11:271-7.

18. Karikari AB, Obiri-Danso K, Frimpong EH, Krogfelt KA. Antibiotic resistance in Campylobacter isolated from patients with gastroenteritis in a Teaching Hospital in Ghana. Open J Med Microbiol. 2017b;7:1-11.

19. Jouahri M, Asehraou A, Karib H, Hakkou A, Touhami M. Prevalence and control of thermotolerant Campylobacter species in raw poultry meat in Morocco. MESO. 2007;9:262-7.

20. Nzouankeu A, Ngandjio A, Ejenguele G, Njine T, Wouafo MN. Multiple contaminations of chickens with Campylobacter, Escherichia coli and Salmonella in Yaounde, Cameroon. J Infect Dev Ctries. 2010;4:583-6.

21. Wieczorek K, Szewczy KR, Osek J. Prevalence, antimicrobial resistance and molecular characterization of Campylobacter jejuni and Campylobacter coli isolated from retail raw meat in Poland. Vet Med. 2012;57(6):293-9.

22. Asuming-Bediako N, Parry-Hanson Kunadu A, Abraham S. Habib I. Campylobacter at the humanfood interface: The African perspective. Pathogens. 2019;8(2):87.

23. Hassanain NA. Antimicrobial resistant Campylobacter jejuni isolated from humans and animals in Egypt. Glob Vet. 2011;6:195-200.

24. Komba EVG, Mdegela RH, Msoffe PLM, Nielsen LN, Ingmer H. Prevalence, antimicrobial resistance and risk factors for thermophilic Campylobacter infections in symptomatic and asymptomatic humans in Tanzania. Zoonoses Public Health. 2015;62:557-68.

25. Nwankwo IO, Faleke OO, Salihu MD, Magaji AA, Musa U, Garba J. Epidemiology of Campylobacter species in poultry and humans in the four agricultural zones of Sokoto State, Nigeria. J Public Health Epidemiol. 2016;8:184-90.

26. Megraud F, Gavinet AM, Camou-Junca C. Serogroups and biotypes of human strains of Campylobacter jejuni and Campylobacter coli isolated in France. Eur J Clin Microbiol Infect Dis. 
1987;6:641-5.

27. Baserisalehi M, Bahador N, Kapadnis BP. Isolation and characterization of Campylobacterspp. from domestic animals and poultry in south Iran. Pak J Biol Sci. 2007;10(9):1519-24.

28. Salihu MD, Junaidu AU, Magaji AA, Abubakar MB, Adamu AY, Yakubu AS. Prevalence of Campylobacter in poultry meat in Sokoto Northwestern Nigeria. J Public Health Epidemiol. 2009;1(2):41-5.

29. Nadeau E, Messier S, Quessy S. Comparison of Campylobacter isolates from poultry and humans: Association between in vitro virulence properties, biotypes, and Pulsed-Field Gel Electrophoresis Clusters. Appl Environ Microbiol. 2003;69(10):6316-20.

30. Engberg J, Aarestrup FM, Taylor DE, Gerner-Smidt P, Nachamkin I. Quinolone and macrolide resistance in Campylobacter jejuni and C. coli: resistance mechanisms and trends in human isolates. Emerg Infect Dis. 2001;7:24-34.

31. Osterlund A, Hermann M, Kahlmeter G. Antibiotic resistance among Campylobacter jejuni/coli strains acquired in Sweden and abroad: A longitudinal study. Scand J Infect Dis. 2003;35(8):478-81.

32. Abamecha A, Assebe G, Tafa B, Wondafrash B. Prevalence of thermophilic Campylobacter and their antimicrobial resistance profile in food animals in Lare District, Nuer Zone, Gambella, Ethiopia. J Drug Res Dev. 2015;1(2).

33. Okunlade A, Ogunleye AO, Jeminlehin FO, Ajuwape AT. Occurrence of Campylobacter species in beef cattle and local chickens and their antibiotic profiling in Ibadan, Oyo State, Nigeria. Afr J Microbiol Res. 2015;9(22):1473-9.

34. Helms M, Simonsen J, Olsen KE, Mølbak K. Adverse health events associated with antimicrobial drug resistance in Campylobacter species: A registry-based cohort study. J Infect Dis. 2005;191(7):10505 .

\section{Figures}




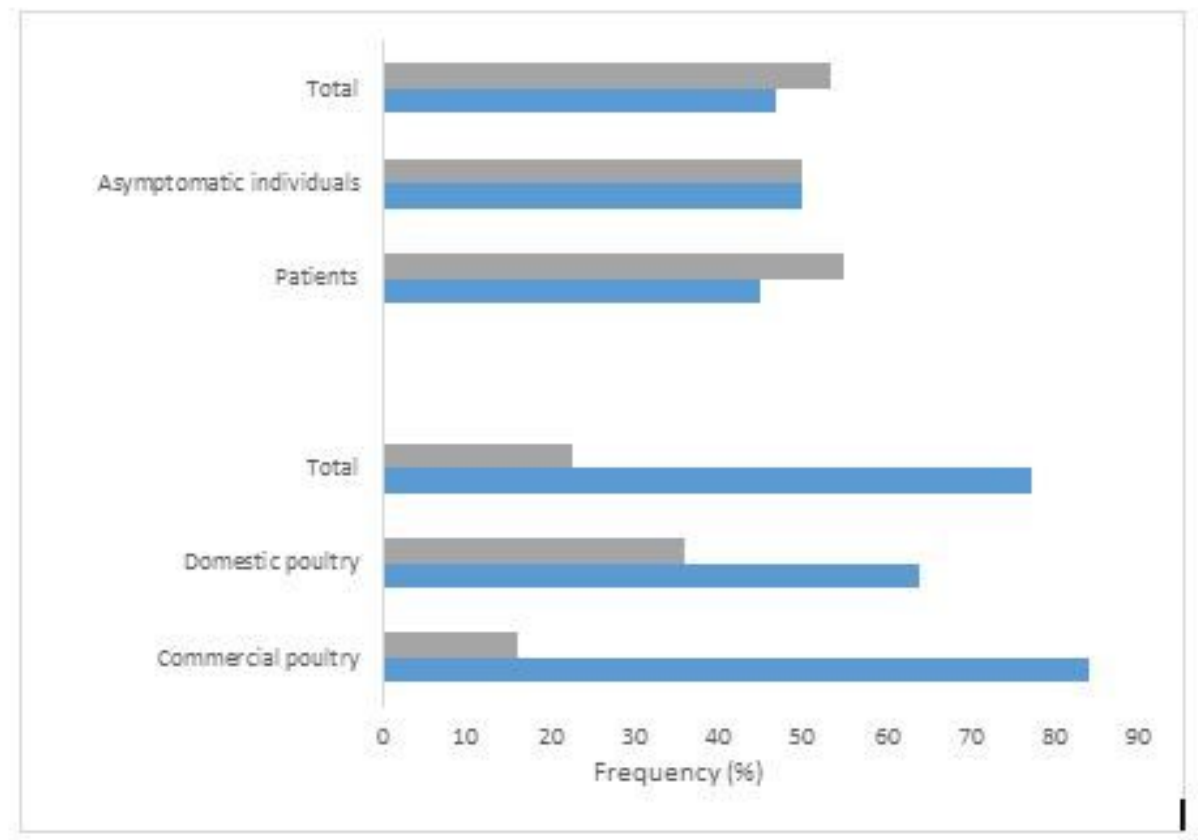

Figure 1

C. jejuni non-jejuni

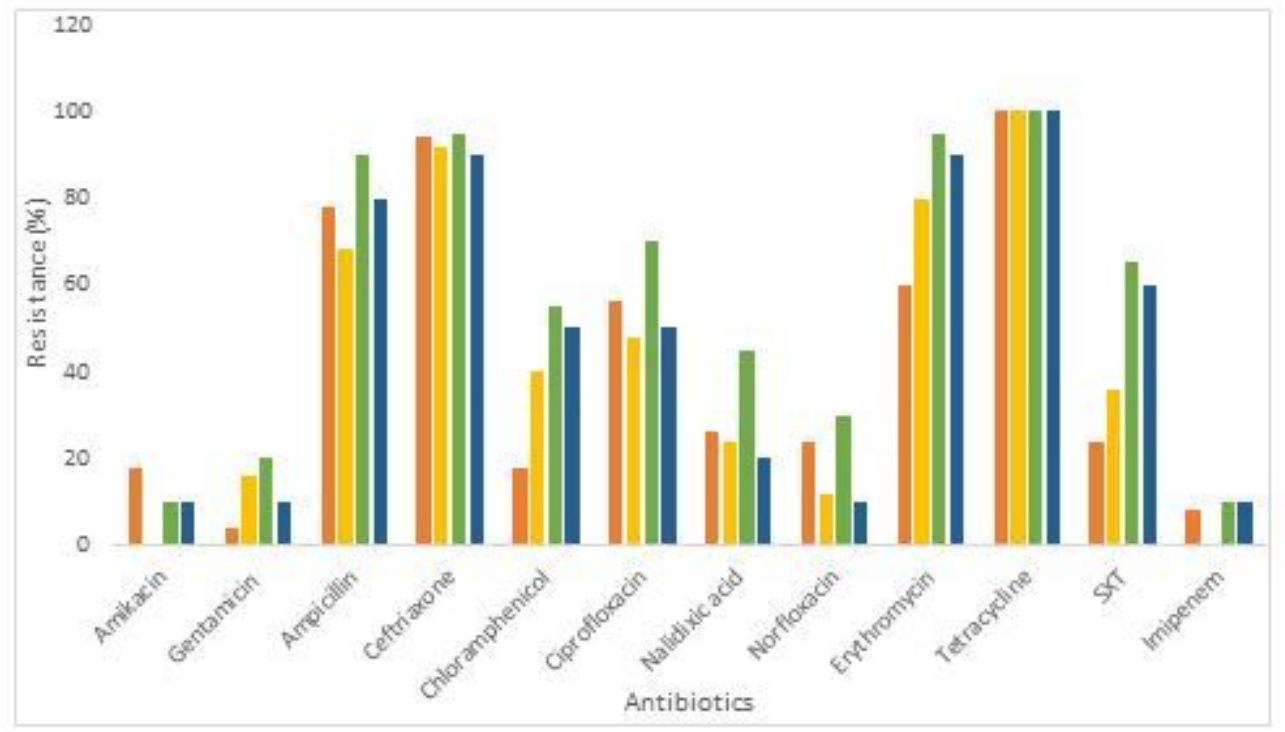

Figure 2

Commercial poultry $n=50$ Domestic poultry $n=25$ Patients $n=20$ Asymptomatic individuals $n=10$ 


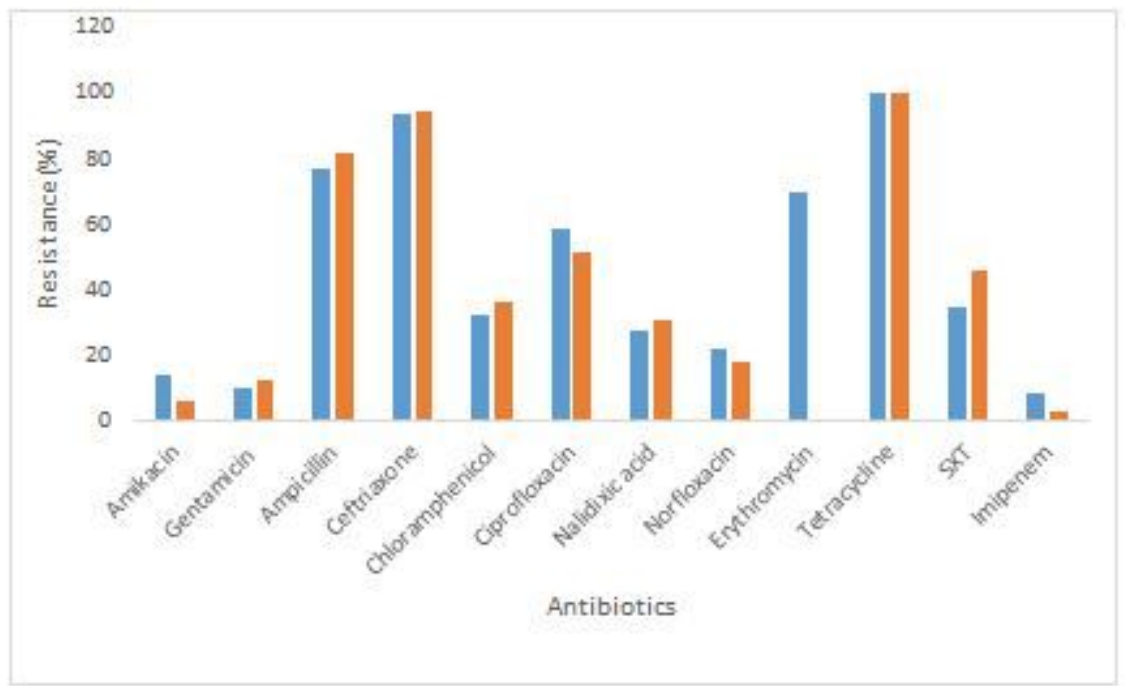

Figure 3

C. jejuni $n=72$ non-jejuni $n=33$

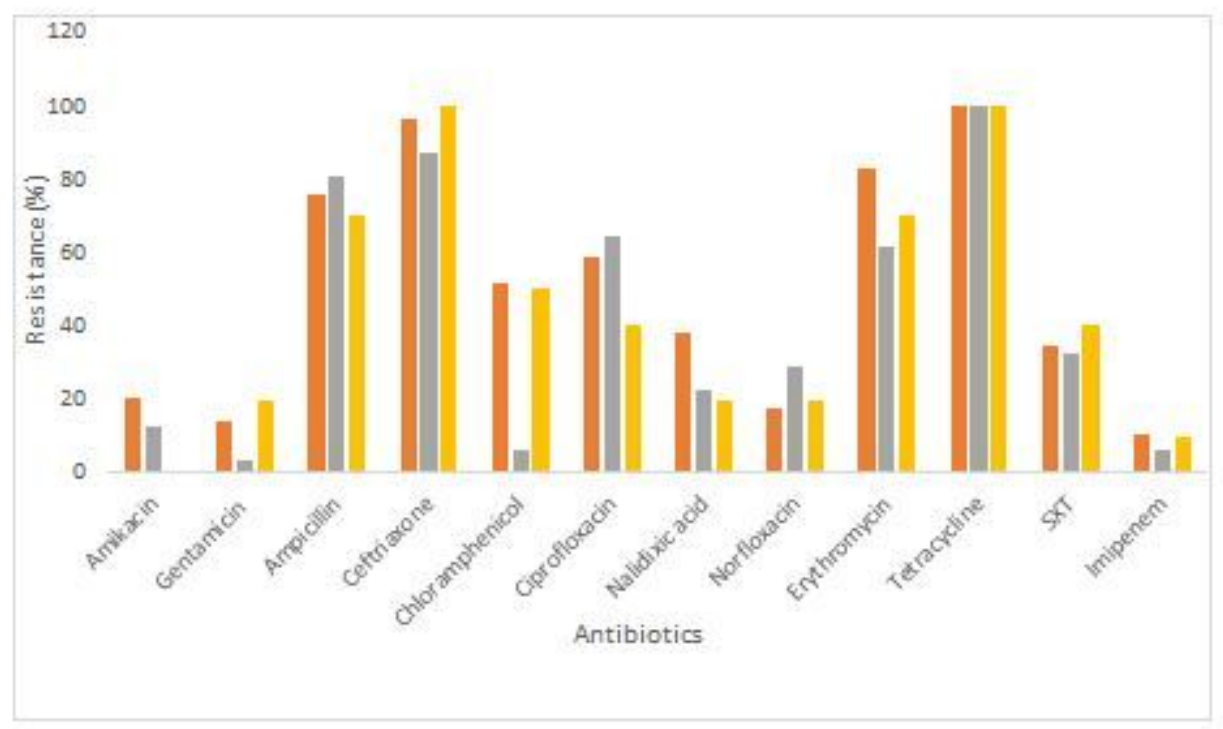

Figure 4

Biotype I $\mathrm{n}=29$ Biotype II $\mathrm{n}=31$ Biotype III $\mathrm{n}=10$ 